\title{
Malignant peripheral nerve sheath tumors of the spine: results of surgical management from a multicenter study
}

\author{
Dean Chou, MD, ${ }^{1}$ Mark H. Bilsky, MD, ${ }^{2}$ Alessandro Luzzati, MD, ${ }^{3}$ Charles G. Fisher, MD, MHSc, ${ }^{4}$ \\ Ziya L. Gokaslan, MD, ${ }^{5}$ Laurence D. Rhines, MD, ${ }^{6}$ Mark B. Dekutoski, MD, ${ }^{7}$ \\ Michael G. Fehlings, MD, ${ }^{8}$ Ravi Ghag, MD, ${ }^{4}$ Peter Varga, MD, ${ }^{9}$ Stefano Boriani, MD, ${ }^{10}$ \\ Niccole M. Germscheid, MSc, ${ }^{11}$ Jeremy J. Reynolds, MBChB, ${ }^{12}$ and \\ the AOSpine Knowledge Forum Tumor
}

1Department of Neurosurgery, University of California, San Francisco, California; ${ }^{2}$ Department of Neurosurgery, Memorial Sloan Kettering Cancer Center, New York, New York; ${ }^{3}$ Department of Orthopedics, Istituto Ortopedico Galeazzi, Milan, Italy; ${ }^{4}$ Department of Orthopaedics, University of British Columbia, Vancouver, Canada; ${ }^{5}$ Department of Neurosurgery, Brown University, Providence, Rhode Island; ${ }^{6}$ Department of Neurosurgery, MD Anderson Cancer Center, Houston, Texas; ${ }^{7}$ Department of Orthopedics, The CORE Institute, Phoenix, Arizona; ${ }^{8}$ Department of Neurosurgery, University of Toronto, Ontario, Canada; ${ }^{9}$ Department of Orthopedics, National Center for Spinal Disorders, Budapest, Hungary; ${ }^{10}$ Department of Orthopedics, The Rizzoli Institute, Bologna, Italy; ${ }^{11} \mathrm{AOSp}$ ine International, Davos, Switzerland; and ${ }^{12}$ Department of Orthopaedics, Oxford University Hospitals, Oxford, United Kingdom

OBJECTIVE Malignant peripheral nerve sheath tumors (MPNSTs) are rare soft-tissue sarcomas. Resection is the mainstay of treatment and the most important prognostic factor. However, complete resection of spinal MPNSTs with tumor-free margins is challenging due to the likelihood of residual tumor cells. The objective of this study was to describe whether the type of Enneking resection in the management of spinal MPNSTs had an effect on local recurrence and survival.

METHODS The AOSpine Knowledge Forum Tumor developed a multicenter database that includes demographic, diagnostic, therapeutic, local recurrence, and survival data on patients with primary spinal column tumors. Patients who had undergone surgery for a primary spinal MPNST were included and were analyzed in 2 groups: 1) those undergoing Enneking appropriate (EA) resections and 2) those undergoing Enneking inappropriate (EI) resections. EA surgery was performed if there was histopathological evidence of an intact tumor pseudocapsule and at least a marginal resection on a vital structure. El surgery was performed if there was an intentional or inadvertent transgression of the margin.

RESULTS Between 1993 and 2012, 29 primary spine MPNSTs were identified in 12 (41\%) females and 17 (59\%) males with a mean age at diagnosis of $40 \pm 17$ years (range 5-74 years). The median patient follow-up was 1.3 years (range 42 days to 11.2 years). In total, 14 (48\%) patients died and $14(48 \%)$ patients suffered a local recurrence, $10(71 \%)$ of whom died. Within 2 years after surgery, the median survival and local recurrence were not achieved. Data about Enneking appropriateness of surgery were available for 27 patients; 9 (33\%) underwent an EA procedure and 18 (67\%) underwent an El procedure. Enneking appropriateness did not have a significant influence on local recurrence or survival. Twenty-two patients underwent adjuvant treatment with combined chemo- and radiotherapy $(n=7)$, chemotherapy alone $(n=3)$, or radiotherapy alone $(n=12)$. Adjuvant therapy had no significant influence on recurrence or survival.

CONCLUSIONS The rates of recurrence and survival were similar for spinal MPNSTs regardless of whether patients had an EA or El resection or received adjuvant therapy. Other factors such as variability of pathologist interpretation, PET CT correlation, or neurofibromatosis Type 1 status may play a role in patient outcome. Nonetheless, MPNSTs should still be treated as sarcomas until further evidence is known. The authors recommend an individualized approach with careful multidisciplinary decision making, and the patient should be informed about the morbidity of en bloc surgery when considering MPNST resection.

https://thejns.org/doi/abs/10.3171/2016.8.SPINE151548

KEY WORDS malignant peripheral nerve sheath tumor; MPNST; multicenter; surgery; survival; outcome; oncology

ABBREVIATIONS AOSKFT = AOSpine Knowledge Forum Tumor; EA = Enneking appropriate; $\mathrm{El}=$ Enneking inappropriate; MPNST = malignant peripheral nerve sheath tumor; NF1 = neurofibromatosis Type 1.

SUBMITTED December 29, 2015. ACCEPTED August 8, 2016.

INCLUDE WHEN CITING Published online November 11, 2016; DOI: 10.3171/2016.8.SPINE151548. 
$\mathrm{M}$ ALIGNANT peripheral nerve sheath tumors (MPNSTs) are rare soft-tissue sarcomas originating from Schwann cells or pluripotent cells of neural crest origin and account for 3\%-10\% of all softtissue sarcomas. ${ }^{14,24}$ The WHO coined the term "malignant peripheral nerve sheath tumor" to replace previous heterogeneous and sometimes misleading terminology, such as "malignant schwannoma," "malignant neurilemmoma," "neurogenic sarcoma," and "neurofibrosarcoma."14,24 The incidence of MPNST in the general population is $0.001 \%$, and it is higher in patients with neurofibromatosis Type 1 (NF1) $(3 \%-5 \%) .{ }^{14}$ In addition to NF1, another important risk factor is prior radiation exposure. A review of the literature has shown that 4\%-11\% of MPNSTs are thought to arise from previously irradiated areas. ${ }^{14}$ MPNSTs can appear either de novo or from sarcomatous degeneration within a preexisting plexiform neurofibroma. ${ }^{14,24}$ They can originate from peripheral nerve branches or sheaths of peripheral nerve fibers, and the most common locations are the trunk, limbs, and head and neck. ${ }^{24}$ Only $2 \%-3 \%$ of all MPNSTs arise from the spinal nerves, and the 5-year survival rate for this location is less than $20 \%$, a poor prognosis because of local recurrence or systemic spread. ${ }^{16}$ Resection of MPNSTs followed by radiation therapy is the standard treatment for extraspinal MPNSTs. ${ }^{13}$ The surgical technique of en bloc resection achieving wide margins is an important prognostic factor in achieving local tumor control. ${ }^{23}$ The role of chemotherapy and the development of biologics are still being defined. ${ }^{3,14,19}$ En bloc resection of spinal MPNST with wide margins can be difficult because of residual tumor cells on such vital structures as the dura, other critical nerves, viscera, or large blood vessels, as well as skip lesions.

The AOSpine Knowledge Forum Tumor (AOSKFT) has developed a multicenter, multinational database to gather information pertaining to a range of primary spinal tumors. By combining data from multiple centers, a higher number of MPNSTs could be analyzed, despite the rarity of this tumor. Although other studies have reported larger numbers of MPNSTs, those numbers either include all peripheral nerves or are generated from administrative databases. ${ }^{12,17,21}$ To our knowledge, we report the highest number of MPNSTs solely arising from the axial skeleton along with individual patient data. The objective of this study was to describe whether the type of Enneking resection in the management of spinal MPNSTs had an effect on local recurrence and survival.

\section{Methods}

Patient data were extracted from the AOSpine Multicenter Primary Spinal Tumor Database, which includes approximately 1500 patients treated between 1981 and 2012 at 13 spine centers across North America, Europe, and Australia. Contributing centers were included based on adequate primary tumor caseload ( $>10$ patients), expertise in histopathology, and a history of excellence in epidemiological and outcome measurements. Each center received institutional review and ethics committee approval prior to data entry. For this study, patients were included if they had been treated surgically for a primary diagnosis of MPNST arising from the spine between 1993 and 2012. Data were compiled via a secure web-based application (REDCap, Vanderbilt University) both prospectively and retrospectively from hospital clinical notes and local databases. The AOSKFT met regularly and addressed areas of concern within the data identified by appointed study coordinators, in addition to direct communication between coordinators and individual centers.

Collected data included demographics, diagnostic data, Enneking staging,, 910 therapeutic data, local recurrence, tumor size, and survival data retrieved cross-sectionally. Recurrence was defined as local tumor reappearance after total resection or tumor growth after subtotal resection on a postoperative MRI.

Surgical treatment for these lesions was defined as either Enneking appropriate (EA) or Enneking inappropriate (EI) based on the premise that an EA resection for an MPNST is en bloc resection with wide or marginal margins and that EI resection for an MPNST is a piecemeal or an intralesional resection. The pathologists' assessment of surgical margins was used as the final arbiter as to whether the procedure had been performed in an EA or EI manner. Those patients who had undergone a previous surgical intervention for their MPNST with resections described as intralesional were also considered to have undergone EI resection in their definitive surgery. MPNST is a malignant lesion, and clinical outcome has been described as being dependent on resection. EA surgery for a high-grade MPNST would therefore be traditionally categorized in the extremity as an en bloc resection to achieve wide margins (i.e., removing the tumor as a single specimen with a cuff of normal tissue). Often, spinal MPNSTs involve several compartments, including nerve, soft tissue, and bone. An EA resection would include en bloc resection with wide margins of each compartment. If the margin had been transgressed either intentionally or inadvertently, the surgery would have been deemed an EI even if the surgical plan had been EA resection. To be deemed an EA resection, both surgical perception regarding intraoperative transgression and histopathological evidence were required.

Descriptive statistics were used to evaluate the patient and tumor data. Cohort differences were analyzed using the Student t-test for continuous variables and Fisher's exact test for categorical variables. The Kaplan-Meier survival curves and log-rank tests were used to analyze time to first local recurrence and survival data over a 2-year postoperative period. Statistical significance was determined as $\mathrm{p} \leq 0.05$. All statistical analyses were performed using Stata (version 12.0, StataCorp).

\section{Results}

Twenty-nine primary spine MPNSTs were identified in $12(41 \%)$ females and $17(59 \%)$ males with a mean age at diagnosis of $40 \pm 17$ years (range 5-74 years). The mean tumor dimensions were $7.2 \pm 4.8 \mathrm{~cm}($ range $0.3-20 \mathrm{~cm}) \times$ $5.5 \pm 3.0 \mathrm{~cm}($ range $1.2-13 \mathrm{~cm}) \times 5.0 \pm 3.0 \mathrm{~cm}($ range $0.3-$ $13 \mathrm{~cm}) ; 22(76 \%)$ lesions were located within the mobile spine, and the remaining 7 (24\%) were in the fixed spine. The median patient follow-up was 1.3 years (range 42 days 
TABLE 1. Summary of patient demographic and clinical characteristics

\begin{tabular}{|c|c|}
\hline Variable & Value \\
\hline \multicolumn{2}{|l|}{ Mean age in yrs $(n=29)$} \\
\hline At diagnosis & $40 \pm 17$ \\
\hline At surgery & $41 \pm 17$ \\
\hline \multicolumn{2}{|l|}{$\operatorname{Sex}(n=29)$} \\
\hline Female & $12(41)$ \\
\hline Male & $17(59)$ \\
\hline \multicolumn{2}{|l|}{ Pain at diagnosis $(n=29)$} \\
\hline No & $2(7)$ \\
\hline Yes & $27(93)$ \\
\hline \multicolumn{2}{|c|}{ Pathological fracture at diagnosis $(n=28)$} \\
\hline No & $26(93)$ \\
\hline Yes & $2(7)$ \\
\hline \multicolumn{2}{|c|}{ Previous spine tumor operation $(n=29)$} \\
\hline No & $19(66)$ \\
\hline Yes & $10(34)$ \\
\hline \multicolumn{2}{|l|}{ Method of diagnosis $(n=29)$} \\
\hline Open biopsy & $8(28)$ \\
\hline CT-trocar biopsy & $13(45)$ \\
\hline Intraop biopsy & $8(28)$ \\
\hline \multicolumn{2}{|l|}{ ASIA \& Frankel grade $(n=23)^{\star}$} \\
\hline B & $1(4)$ \\
\hline $\mathrm{D}$ & $7(30)$ \\
\hline$E$ & $15(65)$ \\
\hline \multicolumn{2}{|l|}{ Preop embolization $(n=29)$} \\
\hline No & $22(76)$ \\
\hline Yes & $7(24)$ \\
\hline \multicolumn{2}{|l|}{ Adjuvant therapy $(n=29)$} \\
\hline No & $7(24)$ \\
\hline Yes & $22(76)$ \\
\hline \multicolumn{2}{|c|}{ Timing of chemotherapy $(n=29)$} \\
\hline Preop & $5(17)$ \\
\hline Postop & $1(3)$ \\
\hline Both & $4(14)$ \\
\hline None & $19(66)$ \\
\hline \multicolumn{2}{|c|}{ Timing of radiation therapy $(n=28)$} \\
\hline Preop & $9(32)$ \\
\hline Postop & $7(25)$ \\
\hline Both & $2(7)$ \\
\hline None & $10(36)$ \\
\hline \multicolumn{2}{|c|}{ Local recurrence over the study period $(n=29)$} \\
\hline No & $15(52)$ \\
\hline Yes & $14(48)$ \\
\hline \multicolumn{2}{|l|}{ 2-year local recurrence $(n=29)$} \\
\hline No & $18(62)$ \\
\hline Yes & $11(38)$ \\
\hline \multicolumn{2}{|c|}{ Survival over study period $(n=29)$} \\
\hline Alive & $15(52)$ \\
\hline Dead & $14(48)$ \\
\hline
\end{tabular}

CONTINUED IN NEXT COLUMN "
" CONTINUED FROM PREVIOUS COLUMN

TABLE 1. Summary of patient demographic and clinical characteristics

\begin{tabular}{ll}
\hline \multicolumn{1}{c}{ Variable } & Value \\
\hline 2-yr survival $(\mathrm{n}=29)$ & \\
\hline Alive & $17(59)$ \\
\hline Dead & $12(41)$ \\
\hline
\end{tabular}

ASIA = American Spinal Injury Association.

Values are presented as the number of patients (\%) unless indicated otherwise. Mean values are presented as the mean \pm SD.

* When a discrepancy between American Spinal Injury Association and

Frankel score occurred, the more severe score was chosen.

to 11.2 years). Ten (34\%) patients had a previous spine tumor operation, and procedures in 8 of these patients (80\%) were reported as intralesional resections, thus considered EI procedures. Patient demographic and clinical characteristics are summarized in Table 1.

\section{Enneking Appropriateness}

Data about Enneking appropriateness of surgery were available for 27 patients; 9 (33\%) received an EA procedure, and $18(67 \%)$ received an EI procedure. Clinical characteristics for each cohort of Enneking appropriateness are outlined in Table 2, and there were no significant differences between the cohorts.

\section{Local Recurrence}

In total, 14 (48\%) patients suffered a local recurrence. Within 2 years after surgery, the median local recurrence was not achieved. Considering only those patients for whom we have data available for Enneking appropriateness, $5(56 \%)$ of 9 EA patients and $8(44 \%)$ of 18 EI patients suffered a local recurrence (Table 2). Kaplan-Meier analysis conducted over a 2-year period following resection demonstrated no difference between the EA and EI cohorts for time to first local recurrence $(\mathrm{p}=0.385$; Fig. 1$)$.

\section{Survival}

Fourteen (48\%) patients died during the study period. Within 2 years after surgery, the median survival for MPNST patients was not achieved. Considering only those patients for whom we have data available for Enneking appropriateness, $4(44 \%)$ of 9 EA patients and $9(50 \%)$ of 18 EI patients died (Table 2). Kaplan-Meier analysis conducted over a 2-year period following resection demonstrated no difference between the EA and EI cohorts for survival $(\mathrm{p}=0.962$; Fig. 2).

\section{Adjuvant Therapy}

Twenty-two patients underwent adjuvant treatment with combined chemo- and radiotherapy $(\mathrm{n}=7)$, chemotherapy alone $(n=3)$, or radiotherapy alone $(n=12)$. Adjuvant therapy had no significant influence on recurrence or survival.

\section{Discussion}

To our knowledge, this study represents the largest se- 
TABLE 2. Study characteristics according to Enneking appropriateness

\begin{tabular}{|c|c|c|c|}
\hline Variable & $\mathrm{EA}(\mathrm{n}=9)$ & El $(n=18)$ & $p$ Value \\
\hline \multicolumn{4}{|l|}{ Mean age in yrs } \\
\hline At diagnosis & $38.9 \pm 16.3$ & $40.1 \pm 18.5$ & $0.874^{*}$ \\
\hline At surgery & $40.4 \pm 17.1$ & $41.3 \pm 18.5$ & $0.899^{*}$ \\
\hline Sex & & & $0.683 \dagger$ \\
\hline Female & $3(33)$ & $9(50)$ & \\
\hline Male & $6(67)$ & $9(50)$ & \\
\hline Method of diagnosis & & & $0.227 \dagger$ \\
\hline Open biopsy & $1(11)$ & $7(39)$ & \\
\hline CT-trocar biopsy & $6(67)$ & $6(33)$ & \\
\hline Intraop biopsy & $2(22)$ & $5(28)$ & \\
\hline Spinal location & & & $0.136 \dagger$ \\
\hline Mobile & $5(56)$ & $16(89)$ & \\
\hline Fixed & $4(44)$ & $2(11)$ & \\
\hline $\begin{array}{l}\text { No. of vertebral levels } \\
\text { spanned by tumor }\end{array}$ & & & $0.364 \dagger$ \\
\hline 1 & $3(33)$ & $6(33)$ & \\
\hline 2 or 3 & $3(33)$ & $10(56)$ & \\
\hline$\geq 4$ & $3(33)$ & $2(11)$ & \\
\hline Tumor grade & & & $0.333 \dagger$ \\
\hline Low (I) & $1(11)$ & $0(0)$ & \\
\hline High (II) & $8(89)$ & $18(100)$ & \\
\hline Adjuvant therapy & & & $0.367 \dagger$ \\
\hline No & $3(33)$ & $3(17)$ & \\
\hline Yes & $6(67)$ & $15(83)$ & \\
\hline $\begin{array}{l}\text { Local recurrence over study } \\
\text { period }\end{array}$ & & & $0.695 \dagger$ \\
\hline No & $4(44)$ & $10(56)$ & \\
\hline Yes & $5(56)$ & $8(44)$ & \\
\hline 2-yr local recurrence & & & $0.683 \dagger$ \\
\hline No & $5(56)$ & $12(67)$ & \\
\hline Yes & $4(44)$ & $6(33)$ & \\
\hline Survival over study period & & & $1.000 \dagger$ \\
\hline Alive & $5(56)$ & $9(50)$ & \\
\hline Dead & $4(44)$ & $9(50)$ & \\
\hline 2-yr survival & & & $1.000 \dagger$ \\
\hline Alive & $5(56)$ & $10(56)$ & \\
\hline Dead & $4(44)$ & $8(44)$ & \\
\hline
\end{tabular}

Values are presented as the number of patients $(\%)$ unless indicated otherwise. Mean values are presented as the mean \pm SD.

* Student t-test.

$\dagger$ Fisher's exact test.

$\ddagger$ WHO grades.

ries of MPNSTs arising solely from the spine and with individual data collected from centers (as opposed to an administrative database) to date. There are other larger series in which extremity or axial MPNSTs are discussed, but the anatomical constraints of spinal MPNSTs preclude generalization from papers that also include extremity MPNSTs; however, the paradigms developed for nonspinal MPNSTs can be extrapolated to spinal lesions.

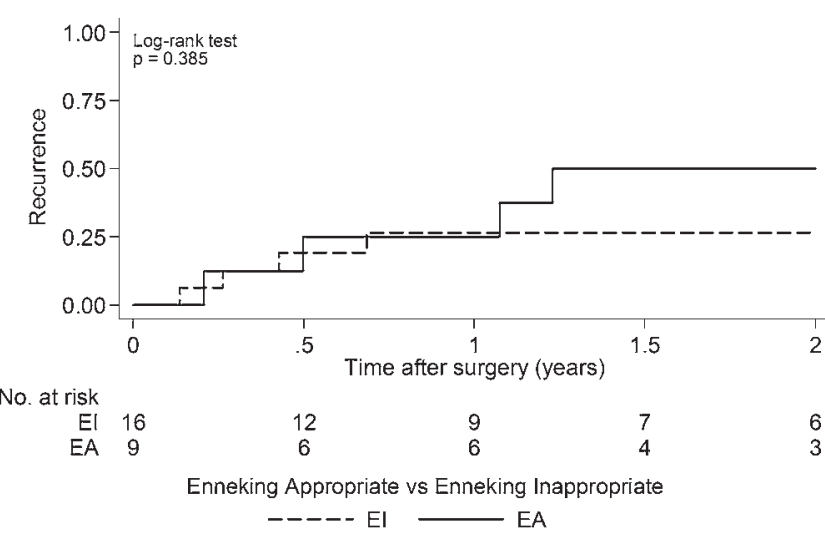

FIG. 1. Kaplan-Meier analysis of first time to local recurrence over a 2-year postoperative period comparing EA versus El resections. The number at risk indicates the number of patients included in the analysis. Information regarding EA/El and timing of local recurrence is known for 25 patients. Median local recurrence was reached at 1.2 years postoperatively for EA patients and was not reached for El patients during this period.

Indeed, spinal MPNSTs have previously been described as having a significantly poorer outcome than their nonspinal equivalent. ${ }^{4,14}$ Survival in patients undergoing EA and EI resections in our series (56\% and $50 \%$, respectively) has improved over that previously reported $(20 \%) \cdot{ }^{16,19}$ More modern studies also report higher survival rates; however, factors that may have influenced the higher survival rates seen in our series compared with historical controls may be related to the tertiary care nature of the centers contributing data or to improved modern surgical management.

Stadler et al. evaluated an administrative database and reported on 64 patients with MPNST; ${ }^{21}$ the 5 -year survival rate was $65 \%$. Although they reported higher numbers of patients, the granularity of that data set is limited because it arises from an administrative database. Vital factors such as pathological confirmation, tumor size, and type of surgery cannot be garnered from an administrative database. Moreover, descriptive coding of the diagnosis and the procedure performed are examples of details that are largely reliant on a coder, not the surgeon. The reliance on a coder may induce inaccuracies in reporting. For instance, in their study, Stadler and coauthors found that patients who underwent radiation therapy and surgery had a higher rate of mortality than patients who underwent surgery alone. Although it does not make clinical sense that radiation therapy would induce higher mortality, such a finding may be the result of a selection bias that could not be further elucidated because of absent data. In this series, more aggressive tumors with significant spread, piecemeal resection, or vital structure invasion may have been selectively biased to receive radiation therapy. Thus, although an administrative database can provide high numbers of patients to study, lack of granularity, reliance on coders, and inability to obtain detailed clinical information limit detailed interpretation.

In our study, we did not find a difference in recurrence rate or survival based on EI or EA resection. While considering MPNST as a sarcoma, one would expect that with an EA resection, there would be better progression-free 


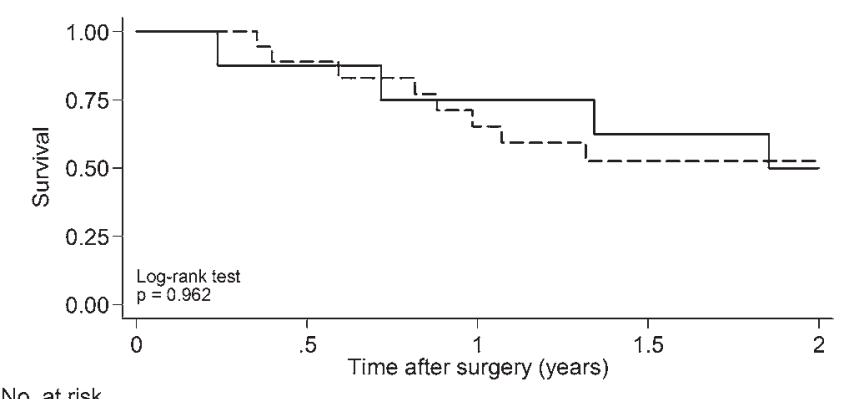

No. at risk

\begin{tabular}{cccccc} 
EA & 9 & 7 & 6 & 5 & 4 \\
EI & 18 & 15 & 11 & 7 & 6 \\
& \multicolumn{5}{c}{ Enneking Appropriate vs Enneking Inappropriate } \\
\cline { 2 - 3 } & EA & $-0---$ El
\end{tabular}

FIG. 2. Kaplan-Meier analysis of survival over a 2-year postoperative period comparing EA versus El resections. The number at risk indicates the number of patients included in the analysis. Information regarding $\mathrm{EA} / \mathrm{El}$ is known for 27 patients. Median survival was reached at 1.9 years postoperatively for EA patients and was not reached during this period for El patients.

survival but not necessarily a benefit in overall survival. ${ }^{23}$ An EA resection for an MPNST is defined as an en bloc resection that achieves wide margins. The most probable explanation for equivalent local control in the EI and EA cohorts is that the biology and anatomy of the tumor precludes obtaining wide margins even when an en bloc resection is performed. Even with histologically negative margins, wide resections of MPNSTs are complicated by the spread along nerves outside of the gross tumor and the presence of multiple skip lesions. For this reason, the resection of an extremity or plexus MPNST by convention involves en bloc resection with a $2-\mathrm{cm}$ margin of the nerve(s) adjacent to the gross tumor. Additionally, spinal MPNSTs often involve several compartments in addition to the nerve, such as epidural and intradural space, bone, and soft tissue. This multicompartment invasion significantly complicates the ability to achieve wide margins and in turn defines microscopic spread compared with nonspinal MPNSTs. While en bloc resection is technically feasible in some patients, the ability to truly achieve a wide margin is restricted by at-risk structures, such as the spinal cord, dura, major blood vessels, and the esophagus. Additionally, the expected survival benefit in the EA group may be adversely impacted by the increased morbidity associated with these procedures compared with EI, specifically intralesional piecemeal resections.

Given the constraints of achieving en bloc resection to achieve wide margins, adjuvant radiation therapy is critically important to consider in these patients. ${ }^{13}$ In our series, more patients received radiation therapy in the EA group than the EI group (83\% vs $67 \%$, respectively). This may in part explain the failure to show benefit in the EA compared with the EI group. In studies examining the outcomes of nonspinal MPNSTs, radiation has demonstrated a significantly improved progression-free survival but not overall survival..$^{23}$ The doses required to treat sarcomas in conventional fractions (1.8-2 Gy) are greater than 60-70 Gy. High-dose delivery to spinal MPNSTs requires either proton beam therapy or specialized photon delivery systems, such as image-guided intensity-modulated radia- tion therapy or volumetric arc therapy.,22 More recently, spine stereotactic radiosurgery has been used to treat spinal sarcomas. Two studies have examined the use of stereotactic radiosurgery as definitive therapy for primary and metastatic tumors. Folkert et al. reviewed 88 patients with 120 metastatic sarcoma lesions. ${ }^{11}$ The 12 -month local control was superior in those receiving high-dose singlefraction (median dose 24 Gy) compared with high-dose hypofractionated radiation (median 28.5 Gy in 3-6 fractions) $(90.8 \%$ vs $84.1 \%$, respectively $[p=0.007]) .{ }^{11}$ Chang et al. reported the outcomes of 27 patients treated for 32 spine sarcomas with doses ranging from 16 to 45 Gy in 1-3 fractions with a median single fraction dose of 21.8 Gy. ${ }^{6}$ Local control rates were reported to be $96.7 \%$ at 6 months, $78.3 \%$ at 1 year, and $76.9 \%$ at 2 years. ${ }^{6}$ Both radiation dose and volume were associated with radiographic control at 2 years. ${ }^{6}$ High-dose conventional fractionated radiation therapy and stereotactic radiosurgery are effective for treating spinal sarcomas, including MPNSTs. The optimal strategy of integrating radiation therapy is currently unknown. The question remains whether radiation therapy should be offered as a pre- or postoperative adjuvant. Additionally, a major concern is the ability to define the microscopic spread of tumor and skip lesions that are the hallmarks of MPSNTs.

Another factor that may help explain the failure of EA to achieve improved outcomes over EI resections is the inconsistency of the reporting of histological grades and the pathologists' interpretations among institutions. A cellular schwannoma has some pathological features of MPNST in terms of having increased mitotic figures, but it may clinically behave very distinctly. ${ }^{1,2,20}$ If the pathologist's evaluation noted one area of the specimen that appeared to be an MPNST but the specimen was actually a cellular schwannoma, a higher survival rate despite a local recurrence could be explained. Moreover, pathologists do not always agree on the diagnosis; what may seem a cellular schwannoma to one pathologist may be classified as an MPNST by another since one factor that is used is the number of mitotic figures per high-power field. The criteria to distinguish between cellular schwannoma and MPNST are based on a spectrum of mitotic figures per high-power field, and this is not universally agreed on by all pathologists. ${ }^{1,2}$ Thus, if one pathologist grades a cellular schwannoma as an MPNST and another pathologist grades it as a cellular schwannoma, an EA resection of a cellular schwannoma may not necessarily improve survival compared with an EI resection. If such patients were included in our study, this may be a reason the survival data did not show a difference.

One of the difficulties with interpreting outcomes with MPNST is the point at which the lesion shifts from benign to malignant. Eventually, this shift may be determined by genetic markers, but currently and within our series, it relies on an individual pathologist's determination of mitotic figures per high-power field. The diagnoses of a cellular schwannoma and low-grade MPNST therefore may overlap, yet the outcomes resulting from EA and EI surgery are expected to differ between cellular schwannoma and MPNST. Core biopsies will also be open to interpretation, and sampling error as cellularity might vary through the 


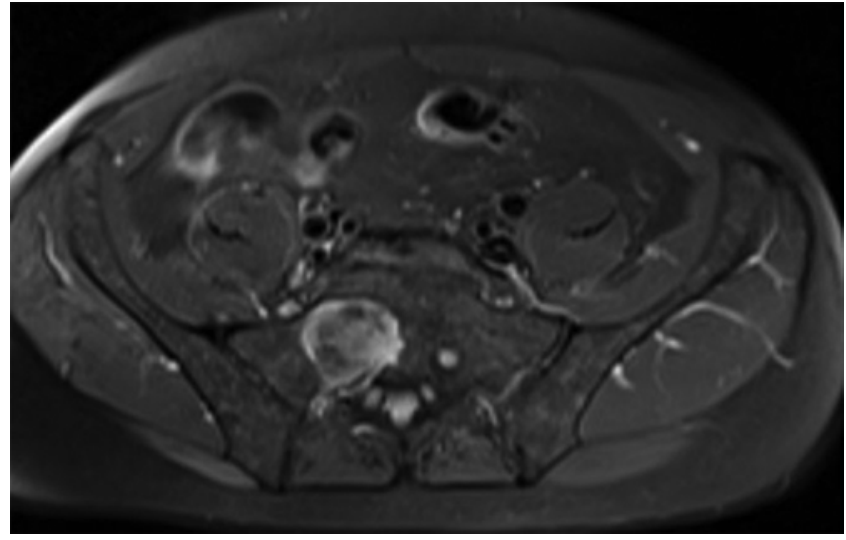

FIG. 3. Axial MR image of the sacrum with contrast demonstrating an example of an S-1 MPNST.

specimen. Planning potentially highly ablative surgery associated with morbidity based on such a result poses a significant clinical conundrum. In addition, given the nature of the nervous structure involved, one cannot rely on a pathologist to truly comment on margins. Microfilament branches from the primarily affected nerve may well carry tumor to distant sites. Such pathways for tumor spread would be impossible to detect preoperatively with current imaging techniques, intraoperatively by the surgeon, or by the pathologists because skip lesions may occur.

Another confounding variable in this study is NF1. This may be a confounding variable in 2 ways. Because many MPNSTs in NF1 patients may be in plexiform neurofibroma bundles, growth after resection might represent continued growth secondary to NF1, not necessarily growth secondary to MPNST. This would account for a longer survival despite what would be called a local "recurrence." By the same token, MPNSTs in patients with NF1 have a much worse prognosis than those who do not have NF1, thus potentially decreasing survival in this subset of the population. ${ }^{8}$ Thus, despite an EA resection in patients with NF1 who have an MPNST, the aggressive nature of MPNST in patients with NF1 may induce the MPNST to spread much more quickly and extensively beyond the confines of what is seen on MRI. Moreover, MPNST in NF1 patients may be much more difficult to clearly resect because of the multiple tumors present in the field. Thus, there may have been a variable associated with NF1 status that affected survival, and this could have lowered the survival rate in patients with NF1 despite EA resection. Unfortunately, because of the ambispective nature of data collection and the limited data fields collected, it is unclear how much or how little of our findings were associated with NF1. Nonetheless, the presence or absence of NF1 may have played a confounding role in our observation, but we unfortunately were unable to track this variable. ${ }^{8}$

Another factor that might have affected our outcomes is imaging. The preoperative MRI study may not have accurately represented the extent of atypical activity seen on PET CT (Figs. 3 and 4). It has been shown that PET CT is useful for staging and restaging patients with MPNSTs and that PET CT is more sensitive than whole-body MRI when evaluating NF1 patients for MPNST, ${ }^{7,15,18}$ A PET CT

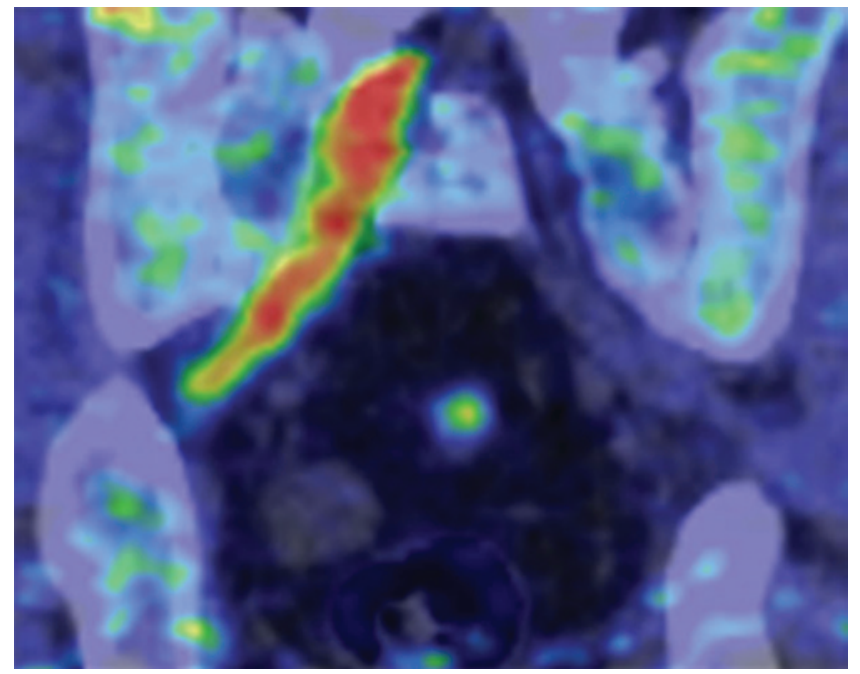

FIG. 4. PET CT image of the MPNST demonstrating the extent of the increased uptake along the course of the S-1 nerve root. Figure is available in color online only.

study may be useful for directing a biopsy toward the more concerning areas of a tumor, and such an area may not have been identified on MRI. Moreover, an increased standardized uptake value on PET CT leads one to be suspicious for MPNST, but one must balance the increased PET CT uptake and the morbidity of en bloc surgery. Additionally, it should be recognized that benign neurofibromas and schwannomas can have markedly elevated standardized uptake values, especially when they are symptomatic. ${ }^{5}$ The balance still lies in determining what degree of morbidity is acceptable while trying to achieve a possibly imperfect oncological result, even with the best planned and well-executed surgery. Unfortunately, in our series, PET CT data were not uniformly available, and we could not make any correlation with PET CT findings, survival, local recurrence, and extent of tumor involvement. For future studies, correlation with MRI, PET CT findings, and extent of tumor involvement will be important factors to address.

There are many limitations to this study. First, the rarity of MPNSTs has resulted in relatively small patient numbers, even with a multicenter, multinational collection data set. Because of these small numbers, the study is underpowered to account for confounding variables and to identify statistically significant changes. Another limitation is the ambispective nature of this study. This limits our ability to have collected more accurate data points about the factors listed earlier, such as NF1 and PET CT status. There was also variability in follow-up. Although many patients died during follow-up, each center may have had different points of follow-up. The length of follow-up was also relatively short in some cases; not all patients reached a follow-up of at least 1 year postoperatively.

Despite not reaching statistical significance for local recurrence or survival with regard to EA versus EI resection, MPNSTs are still currently considered sarcomatous tumors on the spectrum of aggressiveness and, where appropriate, should still be treated as such. The influence of other variables could have played a very pivotal role in 
affecting local recurrence and survival yet could not be further explored in this study. Where technically feasible, en bloc surgery should be considered; however, due to the poor outcomes from both EA and EI surgery, we would not recommend surgery associated with high morbidity in cases in which doubt remains over the pathology or when wide resection is not feasible based on anatomical constraints. High-dose radiation therapy is strongly recommended, regardless of the nature of the resection. In such cases, further studies such as PET CT or genetic testing for NF1 may be considered to provide further confirmation that MPNST is indeed the correct diagnosis.

\section{Conclusions}

In our series, we found that the rates of recurrence and survival were similar for patients regardless of whether they had undergone an EA or EI resection for spinal MPNST. In addition, other factors, such as variability of pathologist interpretation, PET CT correlation, or NF1 status, may have affected survival. Nonetheless, MPNSTs should still be treated as sarcomas until further, more definitive studies can delineate the behavior characteristics of specific MPNSTs. Future studies should consider including such data points as mitotic figures per high-power field, PET CT and MRI correlation, and NF1 status. We would recommend an individualized approach with careful multidisciplinary decision making, and the patient should be informed about the morbidity of en bloc surgery when considering MPNST resection.

\section{Acknowledgments}

We are grateful to the collaborating centers' local clinical research personnel and support staff for their active participation.

\section{References}

1. Alam K, Jain A, Misra A, Khan AH: Cellular schwannoma masquerading as malignant peripheral nerve sheath tumour: a diagnostic dilemma. BMJ Case Rep 2013:bcr2012008435, 2013

2. Alberghini M, Zanella L, Bacchini P, Bertoni F: Cellular schwannoma: a benign neoplasm sometimes overdiagnosed as sarcoma. Skeletal Radiol 30:350-353, 2001

3. Angelov L, Davis A, O'Sullivan B, Bell R, Guha A: Neurogenic sarcomas: experience at the University of Toronto. Neurosurgery 43:56-65, 1998

4. Anghileri M, Miceli R, Fiore M, Mariani L, Ferrari A, Mussi $\mathrm{C}$, et al: Malignant peripheral nerve sheath tumors: prognostic factors and survival in a series of patients treated at a single institution. Cancer 107:1065-1074, 2006

5. Beylergil V, Haque S, Carver A, Bilsky MH, Carrasquillo JA: Schwannomatosis/neurofibromatosis type 2 associated multiple schwannomas visualized on FDG-PET/CT. Rev Esp Med Nucl Imagen Mol 32:275-276, 2013

6. Chang UK, Cho WI, Lee DH, Kim MS, Cho CK, Lee SY, et al: Stereotactic radiosurgery for primary and metastatic sarcomas involving the spine. J Neurooncol 107:551-557, 2012

7. Derlin T, Tornquist K, Münster S, Apostolova I, Hagel C, Friedrich RE, et al: Comparative effectiveness of 18F-FDG PET/CT versus whole-body MRI for detection of malignant peripheral nerve sheath tumors in neurofibromatosis type 1 . Clin Nucl Med 38:e19-e25, 2013

8. Doorn PF, Molenaar WM, Buter J, Hoekstra HJ: Malignant peripheral nerve sheath tumors in patients with and without neurofibromatosis. Eur J Surg Oncol 21:78-82, 1995

9. Enneking WF: A system of staging musculoskeletal neoplasms. Clin Orthop Relat Res (204):9-24, 1986

10. Enneking WF, Spanier SS, Goodman MA: A system for the surgical staging of musculoskeletal sarcoma. Clin Orthop Relat Res (153):106-120, 1980

11. Folkert MR, Bilsky MH, Tom AK, Oh JH, Alektiar KM, Laufer I, et al: Outcomes and toxicity for hypofractionated and single-fraction image-guided stereotactic radiosurgery for sarcomas metastasizing to the spine. Int J Radiat Oncol Biol Phys 88:1085-1091, 2014

12. Gachiani J, Kim D, Nelson A, Kline D: Surgical management of malignant peripheral nerve sheath tumors. Neurosurg Focus 22(6):E13, 2007

13. Gupta G, Mammis A, Maniker A: Malignant peripheral nerve sheath tumors. Neurosurg Clin N Am 19:533-543, v, 2008

14. Gupta G, Maniker A: Malignant peripheral nerve sheath tumors. Neurosurg Focus 22(6):E12, 2007

15. Khiewvan B, Macapinlac HA, Lev D, McCutcheon IE, Slopis JM, Al Sannaa G, et al: The value of ${ }^{18} \mathrm{~F}-\mathrm{FDG}$ PET/CT in the management of malignant peripheral nerve sheath tumors. Eur J Nucl Med Mol Imaging 41:1756-1766, 2014

16. Kourea HP, Bilsky MH, Leung DH, Lewis JJ, Woodruff JM: Subdiaphragmatic and intrathoracic paraspinal malignant peripheral nerve sheath tumors: a clinicopathologic study of 25 patients and 26 tumors. Cancer 82:2191-2203, 1998

17. Okada M, Kitagawa M, Shibuya H, Kanayama K, Sato T, Yamamura H, et al: Malignant peripheral nerve sheath tumor arising from the spinal canal in a cat. J Vet Med Sci 69:683686, 2007

18. Otsuka H, Graham MM, Kubo A, Nishitani H: FDG-PET/CT findings of sarcomatous transformation in neurofibromatosis: a case report. Ann Nucl Med 19:55-58, 2005

19. Prieto R, Pascual JM, García-Cabezas MA, López-Barea F, Barrios L, González-Llanos F: Low-grade malignant triton tumor in the lumbar spine: a rare variant of malignant peripheral nerve sheath tumor with rhabdomyoblastic differentiation. Neuropathology 32:180-189, 2012

20. Santi R, Franchi A, Veltri M, Valeri A, Nesi G: Meningothelial-like whorls in a retroperitoneal cellular schwannoma: potential diagnostic pitfall. Pathol Int 60:62-63, 2010

21. Stadler JA III, Qadri U, Tang JA, Scheer JK, Melkonian SC, Smith ZA, et al: Malignant peripheral nerve sheath tumors of the spine: a SEER database analysis. J Clin Neurosci 21:1106-1111, 2014

22. Terezakis SA, Lovelock DM, Bilsky MH, Hunt MA, Zatcky J, Yamada Y: Image-guided intensity-modulated photon radiotherapy using multifractionated regimen to paraspinal chordomas and rare sarcomas. Int J Radiat Oncol Biol Phys 69:1502-1508, 2007

23. Vauthey JN, Woodruff JM, Brennan MF: Extremity malignant peripheral nerve sheath tumors (neurogenic sarcomas): a 10-year experience. Ann Surg Oncol 2:126-131, 1995

24. Zhu B, Liu X, Liu Z, Yang S, Liao HI, Jiang L, et al: Malignant peripheral nerve sheath tumours of the spine: clinical manifestations, classification, treatment, and prognostic factors. Eur Spine J 21:897-904, 2012

\section{Disclosures}

This study was funded by AOSpine International, through the AOSpine Knowledge Forum Tumor. AOSpine is a clinical division of the AO Foundation - an independent medically guided nonprofit organization. The AOSpine Knowledge Forums are pathology-focused working groups acting on behalf of AOSpine in their domain of scientific expertise. Each forum consists of 
a steering committee of up to 10 international spine experts who meet on a regular basis to discuss research, assess the best evidence for current practices, and formulate clinical trials to advance spine care worldwide. Study support is provided directly through AOSpine's Research Department and the AO Clinical Investigation and Documentation unit. The authors report the following. Dr. Chou: consultant for Globus, Medtronic, and Orthofix. Dr. Fisher: consultant for Medtronic and NuVasive; grant paid to institution by OREF; and royalties from Medtronic. Dr. Rhines: consultant for Stryker and Globus. Dr. Dekutoski: Mayo Office of Intellectual Property, Medtronic Percutaneous fixation. Ms. Germscheid: employed by AOSpine International.

\section{Author Contributions}

Conception and design: Fisher, Gokaslan, Rhines, Dekutoski, Fehlings, Varga, Boriani. Acquisition of data: Chou, Bilsky, Luzzati,
Fisher, Gokaslan, Rhines, Dekutoski, Fehlings, Varga, Boriani, Germscheid, Reynolds. Analysis and interpretation of data: Chou, Bilsky, Germscheid, Reynolds. Drafting the article: Chou, Bilsky, Ghag, Germscheid, Reynolds. Critically revising the article: Chou, Bilsky, Fehlings, Germscheid, Reynolds. Reviewed submitted version of manuscript: Chou, Bilsky, Ghag, Germscheid, Reynolds. Approved the final version of the manuscript on behalf of all authors: Chou. Statistical analysis: Germscheid. Administrative/technical/material support: Germscheid. Study supervision: Germscheid.

\section{Correspondence}

Dean Chou, Department of Neurosurgery, University of California, San Francisco, 505 Parnassus Ave., Box 0112, San Francisco, CA 94143. email: choud@ neurosurg.ucsf.edu. 